\title{
Iterative Minimum Viable Product Approach to Implementing AI, RPA, and BI Solutions
}

\author{
Rishab Srivastava (https://orcid.org/ 0000-0002-2028-1079) \\ Westcliff University
}

\begin{abstract}
Breakthrough technologies can be considered as exponentially disruptive to organizations across industries within the last few decades of the $21^{\text {st }}$ century, as they have significantly altered the way their business units or customers operate. Artificial Intelligence related cognitive technologies are some of the latest disruptive solutions currently being adopted by organizations. Organizational leaders may feel both the pressure and excitement of adopting such nascent technology quickly and at scale. However, due to organizational knowledge gaps of nascent solutions, transformative large-scale initiatives have a higher risk of negative impact on failure to implement. On the other hand, an iterative approach allows for the implementation to occur in smaller amounts and leaves room for incorporating feedback and lessons learned in future iterations, thus mitigating the risks involved with the undertaking. This article breaks down the nascent field of advanced cognitive technologies into three main categories based on their business use cases: process automation, cognitive insights, and cognitive engagement. It then explores implementing this technology in each of its three categories through the lens of a popular iterative product lifecycle management approach (i.e., the Minimum Viable Product) to reduce the risk of failure or other negative impacts on an organization adopting cognitive solutions.
\end{abstract}

Keywords: agile, artificial intelligence, breakthrough technology, business intelligence, business needs, data analytics, Devops, information technology, iterative implementation, megatrend, minimum viable product, MVP, robotic process automation, RPA, solution implementation, UI, user interface, user interface data science, UX, Waterfall

\section{Iterative Minimum Viable Product Approach to Implementing AI, RPA, and BI Solutions}

The technology sector is innately disruptive in nature (Flavin, 2021). It has penetrated and accelerated change in almost all industries globally. Breakthrough innovations can create megatrends which drive the need for large-scale adoption of new technologies, processes, and governance models. Adoption of transformational megatrends are important for organizations to stay relevant and competitive in permanently changing environments (Peciak, 2016). Furthermore, early adoption provides these organizations with additional benefits and competitive advantages. In the digital landscape, machine learning and data analytics are powering a wave of such groundbreaking technologies. Companies are aiming to achieve a competitive advantage in this new landscape by implementing accurate predictive analytics and workflow automation solutions, such as Artificial Intelligence $(\mathrm{Al})$, business intelligence (BI) and robot process automation (RPA) (Wang et al., 2021). However, these nascent breakthrough technologies have their own risks. In order to successfully reap the rewards of these solutions, organizations must incorporate 
appropriate implementation strategies that mitigate risks of failure.

In response to the growing pressures and excitement in the market to adopt new cognitive technologies, organizational leaders should fight the urge to start their journey by pursuing "moon shot" or ambitious projects, and initially focus on "low hanging fruit" or simple initiatives instead (Ries, 2019). Moon shot projects are those of large scope with numerous objectives that need to be met, paired with a large budget, and possibly, multi-year delivery timelines. Completing them comes with the promise of grand rewards; however, chances of failure are high when compared to iterative approaches instead.

New technology may be implemented in organizations in sequential or iterative ways (Sumrell, 2007). Sequential approaches involve intense periods of defining all the requirements for a product, after which the solution is designed, built, tested and then finally implemented as a whole. Alternatively, an iterative approach involves quick implementation, testing, review, and updates of small amounts of features (Njegus \& Milanov, 2011). Such an approach implementing a software development methodology is where the development team initially focuses on creating a simplified set of software features, which then gains a larger set of features as it progressively gains complexities until completion. There are multiple tried and tested iterative methodologies that drive the implementation of various Information Technology initiatives across industries, such as prototypes, pilot initiatives, proof of concepts and fully functional minimum viable products. However, a core component of these methodologies involves a technical team focused on building a set of pre-determined features which will benefit the end consumers (Denton, 2022). In the first iteration, end users and the development team agree on program scope. Lessons learned in the first iteration of the product development are incorporated into product features. This helps the development team familiarize themselves with various unknown aspects of a new technology in a lowrisk environment and enables end users to adopt and incorporate the new technology into their existing business and operational processes with little friction or negative impact on the rest of their day-to-day activities (Kennedy et al., 2018). One such concept of an iterative approach is to create a Minimum Viable Product (MVP). An MVP implementation is a development technique used to create a fully functional product with just enough features to satisfy a group of early adopting customers, who in turn, provide feedback for future iterations of the project (Ries, 2019). In this article, we review the implementation of new cognitive technologies such as Artificial Intelligence and robot process automation through the lens and framework of the iterative MVP approach.

\section{Implementation Approaches for Introducing} New Technology to an Organization

A popular sequential approach is the waterfall methodology (Afshar et al., 2019). Within this approach, requirements are clearly identified in the beginning and a fixed plan of implementation is incorporated. This fixed plan includes several successive phases which are not revisited once complete. In the beginning phase, the organization collaborates with business partners and end users. This is followed by the development phase, which could last weeks or months. Solution testing is conducted after the entire product has been built as per the pre-determined requirements. This sequential approach is challenging for initiatives that involve information discovery, which are cyclical in nature. Without adequate feedback from the end users over time, or testing while building algorithms, the risk of failure is high. For example, the lack of feedback throughout the process may lead to additional functionalities being built on foundationally weak solutions, such as an impractical user interface or unintentional outcomes from algorithms.

Popular iterative approaches to implementing new cognitive solutions, such as Agile and DevOps methodologies, aim to mitigate some of these risks. The Agile project management framework is an iterative approach to developing new solutions by implementing a small batch of features over time (Saltz \& Shamshurin, 2019). This allows for closer collaboration between developers and end users, leading to enhanced usability of the features rolled out over time. Four core values of the Agile framework are: 1) to prioritize individual interactions over systems and 
processes, 2) to create demonstrable working software rather than robust documentation, 3) to collaborate between developers and product stakeholders over contract negotiations, and 4) respond to change rather than following a rigid plan. Within the Agile framework, developers can quickly make adjustments to the solution by incorporating feedback from users.

The Agile framework emphasizes collaboration between the product management team and the core development teams until all features of the solution have been fully completed (Blankenship et al., 2011). The DevOps iterative framework extends this collaborative environment by including the care, feeding, monitoring, and tweaking of the machine learning solutions. This means that in addition to developing the product, the same team continues to support ongoing upgrades and maintenance of the product, even beyond its successful implementation. This allows for both development and product management teams to create more mature models over time with higher levels of accuracy (Sheil et al., 2020).

One of the main reasons cognitive machine learning and data science initiatives fail is due to poor and unintuitive user interface design choices, which reduces the richness and efficacy of end user interactions (Lotte et al., 2018). The waterfall methodology's rigid, sequential approach can be a hindrance to understanding and mitigating the risks of failure to implement appropriate and relevant user interface solutions. User interface is the primary factor out of the four core factors which need to be considered to ensure a robust interface is available for end users: the user, the task, the computer system, and change over time (Vieira et al., 2020).

The primary need is to understand the variations amongst users, such as their roles, backgrounds, and how they intend to use or interact with the respective systems (Burnett, 2012). For example, a technical user with some level of basic statistical knowledge may be comfortable with raw statistical tables to obtain insights. However, a user with a businesscentric leadership role may need visualizations and other stimuli to provide the necessary information they need to make decisions, such as graphs that highlight core decision-making factors on the screen for them. They may need to be guided throughout the user interface through visuals, menus, or explanations to make the appropriate decisions. Within cognitive solution user interfaces, another challenge is not only maintaining large inter-related data, but also providing end users with intuitive information retrieval abilities (Du et al., 2016). The search features should empathize with the users on how and when they want the information available and presented to them.

These challenges can be overcome by iteratively incorporating three design principles focusing on use cases (users and their corresponding tasks), conducting an empirical measurement of prototype designs (such as what aspects are objectively proven to be helpful to users versus what design decisions are ignored or hamper the user experience), and conducting revisions through a feedback loop mechanism between end users and the development team (Seybold, 2011). The feedback loop mechanism, which is innately a core principle of the iterative implementation approach, will help maximize a shared understanding of three main aspects of cognitive enhancement solutions and their interactions with end users: the main goals for the machine learning/artificial intelligence model and solution, the user population's various characteristics, and the nature of the use cases or tasks that need to be accomplished (Gould \& Lewis, 1985).

\section{Breakdown of New Wave of Cognitive Solutions into Three Categories}

Examining new cognitive solutions through the lens of business use cases enables organizations to adopt an iterative strategy focused on their business needs (Cross \& Fouse, 2005). Applied cognitive products can be broken down into three main categories based on the respective business needs they support: process automation, analytical insights, and cognitive engagement.

Process automation involves the automation of digital and physical tasks (e.g., financial spreadsheets, administrative backoffice tasks such as file record arrangements). Examples of such automation tasks include creating "macros" within Microsoft Excel spreadsheets, which are a set of recorded actions that can be manually re-run by the end- 
users in the future (Fleishman-Mayer et al., 2013). However, RPA provides certain advancements over such previously existing business process automation solutions by having "robots" directly replicate pre-recorded human inputs and compile information from various source systems (Januszewski et al., 2021). They are automatically triggered based on a dynamic set of parameters. It can also include doing a sequence of tasks in multiple different applications, with little to no human intervention. For example, RPA tasks may include compiling data from various messages, emails, and other call center record systems, such as updates to customer addresses, and then updating this information in backend customer record systems. Another example may be for an RPA solution to "read" legal documents to extract relevant information, provisions, and dates automatically, with no human intervention.

The second category, cognitive analytical insights, or business intelligence, leverages computer algorithms to comb through large amounts of data, detect patterns, and then alert end users or respective parties when their attention is needed. For example, the fraud department of a large bank can use a fraud analytics solution to comb through all transactions for a customer within a given time to check if any combination of the data matches a pre-determined list of conditions or patterns (Broadhurst et al., 2011). If the system detects a pattern, the system generates an alert, and the relevant information is sent to respective users and parties to pursue next steps and actions as needed. These patterns may be pre-set in a system by a fraud department's preferences and regulatory requirements or may be tweaked based on self-learning feedback loops over time to reduce false positive alarms on customer behavior. Such cognitive insight applications are being used to conduct jobs that are only possible by computers, since humans will be unable to crunch such large amounts of data at such high speeds (Choi \& Lambert, 2017).

The third category, cognitive engagement, assists with directly interacting with end users to understand their needs and, in return, provide information or services as deemed necessary based on the interaction. These include intelligent chatbots that process natural language (Abdellatif et al., 2020). Another possibility is a recommendation system which a retail company's website would use to automatically present a curated list of products or services to a potential customer based on information the customer provided about themselves and their needs.

\section{The First Step - A Minimum Viable Product Framework}

Within the iterative implementation environment, the Minimum Viable Product framework can help incorporate cognitive technologies into organizations by incrementally implementing small initiatives with limited scope but still providing a positive return on investment (Olsen, 2015). This limited scope shall contain a list of features that need to be implemented to render the cognitive technology functional to an end user. To identify the feature sets of the MVP of cognitive technologies, a series of assessments will need to be made and answered within the organization.

First, business areas that have the most to benefit from implementing cognitive technologies need to be identified (Heller, 2019). These areas typically are parts of an organization that are bottlenecked by the fact that information either does not reach them in time or takes a long time to process. Second, these departments need to be evaluated to identify which cases can benefit from cognitive technologies and provide a substantial rate of return. The use cases should identify how critical it is to address the issue, the level of complexity (both from a business process and technical standpoints) it will take to implement an Al solution, and what benefits the organization will receive after launching the application. After these questions are answered, the list of use cases then need to be further prioritized into those that offer value in the short-term, versus those that offer long-term value (i.e., upon which future cognitive capabilities can be further built upon, over time).

Third, based on the use cases that are short-listed, the appropriate Al technology will need to be gauged to see whether cases meet the business needs, or have constraints. For example, RPA solutions may be able to automate certain redundant tasks, such as invoicing and monthly accounting reports, but 
may slow down the respective financial and accounting systems, leading to an overall negative business impact (Bodek, 2018). Out of the three main categories of cognitive technologies, RPA solutions are the least expensive, simplest to implement, and quickly provide a high return on initial investment (Sobczak, 2021). Based on the use case, MVP solutions can be implemented by simply recording end-to-end human interactions on applicable process flows and then replicating and re-running them with the minimum parameters needed to be input by humans over time. More complex RPA scenarios and alternative flows can be incorporated over time as additional builds beyond the MVP phase.

The MVP approach on cognitive insight applications should initially replace non-business critical use cases so as not to impact daily operations while tweaks are being made on the underlying machine learning algorithms to provide the desired outcomes (Paradkar, 2021). For example, an algorithm can be created to crunch existing big data to identify a shortlist of relevant products and services to cross-sell to a customer or future prospect. This algorithm does not seem to impact business-critical operational infrastructure but still provides value via crossselling. A feedback loop mechanism will be required to gauge the algorithm's performance and to make tweaks over time to pursue desired outcomes (Marathe et al., 2019).

Cognitive engagement applications such as chatbots should pursue a similar trend and be implemented in areas of low risk during an MVP build. Instead of being customer-facing, these chatbots could start by being internally facing to reduce the risk of negative impact from failure (Meyer et al., 2020). For example, an IT help desk chatbot can help address many internal needs for employees, such as ordering workspace gear, requesting access to systems or data, and troubleshooting generic issues such as being locked out of an account. This approach will ensure buggy chatbots can be tweaked over time with limited risk of losing customers. A feedback loop will be crucial here as well to ensure the MVP development team obtains the necessary reviews and suggestions by end users, to make changes to chatbot logic over time.

\section{Conclusion}

New technological concepts should not be a burden for organizations to adopt to maintain a competitive advantage and stay relevant in their respective industries. Adopting an iterative approach, rather than a transformative one, to implement new solutions ensures multiple frequent checkpoints are created between the business and technology teams to stay aligned with the core business needs, which then drive what and how new solutions are incorporated into the business.

Transformative changes that aim to replace individuals or entire departments through Al solutions have a high risk of negative impact due to failure, since the scope and budget for such changes are generally large. Comparatively, iterative changes through MVP initiatives that aim to augment human ability, rather than replace their entire skillsets, have a lower risk of negative impact because only a fraction of an otherwise transformative initiative will be assigned to such projects. Thus, organizational strategy should include adopting iterative changes while navigating the learning curve of new technologies in order to create or maintain a competitive advantage in a transforming industry.

\section{References}

Abdellatif, A., Badran, K., \& Shihab, E. (2020). MSRBot: Using bots to answer questions from software repositories. Empirical Software Engineering, 25(3), 1834-1863. https://doi.org/10.1007/s10664-019-09788-5

Afshar, R., Zhang, Y., Firat, M., \& Kaymak, U. (2019). A reinforcement learning method to select ad networks in waterfall strategy [Conference proceedings]. 11th International Conference on Agents and Artificial Intelligence. https://doi.org/10.5220/0007395502560265

Blankenship, J., Bussa, M., \& Millett, S. (2011). Code review. Pro agile. NET development with scrum, 243-271. https://doi.org/10.1007/978-14302-3534-7 9

Bodek, N. (2018). Just-in-time and automation. Kanban Just-in-Time at Toyota, 6578. https://doi.org/10.1201/9780203749715-4 
Broadhurst, R., Bacon-Shone, J., Bouhours, B., Bouhours, T., \& Kingwa, L. (2011). Business and the risk of crime in China ( $\mathrm{Vol} 3$ ). ANU Press.

http://www.jstor.org/stable/10.2307/j.ctt24h93t?r efregid=search-gateway

Burnett, M. (2012). End-user software engineering and why it matters. End-User Computing, Development, and Software Engineering, 185-201.

https://doi.org/10.4018/978-1-4666-01406.ch009

Choi, T.-M., \& Lambert, J. H. (2017). Advances in risk analysis with big data. Risk

Analysis, 37(8), 1435-1442.

https://doi.org/10.1111/risa.12859

Cross, S. E., \& Fouse, S. (2005). Artificial Intelligence and its application to organizational simulation. Organizational Simulation, 425-446. https://doi.org/10.1002/0471739448.ch15

Denton, J. (2022). Mixing methodologies. Research anthology on innovative research methodologies and utilization across multiple disciplines, 222-242. https://doi.org/10.4018/978-1-6684-3881$\underline{7 . \operatorname{ch} 013}$

Du, C., Luo, A., Yang, H., Hou, W., \& Guo, Y. (2016). An efficient method for rare spectra retrieval in astronomical databases. Publications of the Astronomical Society of the Pacific, 128(961), 1-10.

http://www.jstor.org/stable/10.2307/26659933?re freqid=search-gateway

Flavin, M. (2021). Disruptive innovation, the episteme and technology-enhanced learning in higher education. Promtheus, 37(2), 155-169. http://www.jstor.org/stable/10.13169/prometheus 37.2.0155? refregid=search-gateway

Fleishman-Mayer, L. A., Arena, M. V., \& McMahon, M. E. (2013). An excel tool to assess acquisition program risk. RAND.

http://www.jstor.org/stable/10.7249/j.ctt5vjwd6?r efreqid=search-gateway.

Gould, J. D., \& Lewis, C. (1985). Designing for usability: Key principles and what designers think. Communications of the ACM, 28(3), 300311. https://doi.org/10.1145/3166.3170

Heller, C. H. (2019). Near-term applications of artificial intelligence: Implementation opportunities from modern business practices. Naval War College Review, 72(4), 73-100. http://www.jstor.org/stable/10.2307/26775520?re freqid=search-gateway

Januszewski, A., Kujawski, J., \& BuchalskaSugajska, N. (2021). Benefits of and obstacles to RPA implementation in accounting firms. Procedia Computer Science, 192, 46724680.

https://doi.org/10.1016/i.procs.2021.09.245

Kennedy, S. H., Lam, R. W., Nutt, D. J., \& Thase, M. E. (2018). The basics of project evaluation and lessons learned. https://doi.org/10.1201/b17345

Lotte, F., Jeunet, C., Mladenovic, J., N'Kaoua, B., \& Pillette, L. (2018). A BCl challenge for the signal-processing community: Considering the user in the loop. Signal Processing and Machine Learning for Brain-Machine Interfaces, 143-172. https://doi.org/10.1049/pbce114e ch8

Marathe, A., Jain, P., \& Vyas, V. (2019). Iterative improved learning algorithm for petrographic image classification accuracy enhancement. International Journal of Electrical and Computer Engineering, 9(1), 289-296.

https://co21 wh130-mp02-y-https-www-proquestcom.proxy.lirn.net/scholarly-journals/iterativeimproved-learning-

algorithm/docview/2205381400/se2? accountid $=158986$

Njegus, A., \& Milanov, G. (2011). Qualitative comparison of agile and iterative software development methodologies [Conference proceedings]. 2011 19th Telecommunications Forum (TELFOR. https://doi.org/10.1109/telfor.2011.6143783

Peciak, R. (2016). Megatrends and their implications in the globalised world. Horyzonty Polityki, 7(21), 167-184. https://co21wh130mp02-y-https-www-proquestcom.proxy.lirn.net/scholarlyjournals/megatrends-their-implications- 
globalised-world/docview/1880683917/se2?accountid $=158986$

Ries, E. (2019). The lean startup: How constant innovation creates radically successful businesses. Portfolio Penguin.

Meyer, R, Hobert, S., Masuch, K., \& Schumann, M. (2020). Chatbots at digital workplaces - A grounded-theory approach for surveying application areas and objectives. Pacific Asia Journal of the Association for Information Systems, 12(2), 3.

http://co21wh130.mp02.y.http.dx.doi.org.proxy.lir n.net/10.17705/1pais. 12203

Olsen, D. (2015). The lean product playbook: How to innovate with minimum viable products and rapid customer feedback. Wiley.

Paradkar, S. S. (2021). A framework for modeling non-functional requirements for business-critical systems. International Journal of Innovative Research in Computer Science \& Technology, 9(1), 15-19. https://doi.org/10.21276/ijircst.2021.9.1.3

Saltz, J. S., \& Shamshurin, I. (2019). Achieving agile big data science: The evolution of a team's agile process methodology [Conference proceedings]. 2019 IEEE International Conference on Big Data (Big Data). https://doi.org/10.1109/bigdata47090.2019.9005 $\underline{493}$

Seybold, P. (2011). A best practice example of applying UX principles to product design and development. https://doi.org/10.1571/cs06-16$\underline{11 \mathrm{cC}}$
Sheil, E. B., Ramsgaard, E. M., Tamke, E. M., \& Hanna, E. S. (2020). Design transactions: Rethinking information modelling for a new material age.

http://www.jstor.org/stable/10.2307/i.ctv13xprf6? refregid=search-gateway.

Sobczak, A. (2021). Robotic process automation implementation, deployment approaches and success factors - An empirical study. Entrepreneurship and Sustainability Issues, 8(4), 122-147. https://doi.org/10.9770/jesi.2021.8.4(7)

Soh, J., \& Singh, P. (2020). Machine learning operations. Data Science Solutions on Azure, 259-279. https://doi.org/10.1007/978-1-48426405-8

Sumrell, M. (2007). From waterfall to agile - how does a QA team transition? Agile 2007 (AGILE 2007). https://doi.org/10.1109/agile.2007.29

Vieira, S., Lopez Pinaya, W. H., \& Mechelli, A. (2020). Main concepts in machine learning. Machine Learning, 21-44. https://doi.org/10.1016/b978-0-12-815739$\underline{8.00002-x}$

Wang, L., Zhengchao, L., Liu, A., \& Fei, T. (2021). Artificial intelligence in product lifecycle management. The International Journal of Advanced Manufacturing Technology, 114(3-4), 771-796.

http://co21wh130.mp02.y.http.dx.doi.org.proxy.lir n.net/10.1007/s00170-021-06882-1 